\title{
A case of an accidental ingestion of a toothbrush
}

\author{
G. P. U. P. de Silva, R. M. S. S. B. Rathnayake, M.S. E. Karunadasa
}

Surgical Unit, Base hospital Mahiyangana

Keywords: Upper gastrointestinal; foreign body; toothbrush

\section{Introduction}

Approximately 20\% of upper gastrointestinal foreign bodies are reported among adults. The ingestion could be accidental or intentional to achieve secondary gain. Accidental ingestions of non-food objects are rare and reported in patients with neurological, psychiatric illnesses or alcohol intoxication $[1,2]$.

Approximately $80 \%$ of upper gastrointestinal foreign bodies pass without any intervention. However, stiff objects longer than $5-10 \mathrm{~cm}$ are less likely to pass through the lower oesophagal sphincter and have the tendency to impact one of the physiological narrowings of the oesophagus $[3,5]$. Foreign bodies impacted in the oesophagus for more than 24 hours have the risk of complications including pressure necrosis, perforation, mediastinitis, retropharyngeal abscess and aspiration. Therefore an urgent endoscopic intervention is required.

\section{Case Presentation}

We present a case of a 67 years old man who had an accidental ingestion of a toothbrush while brushing teeth. He admitted to the emergency treatment unit of Base Hospital Mahiyangana 1 hour after the incident. The patient could not withstand the strong involuntary drag applied to the toothbrush while brushing the posterior part of the tongue. He complained of retrosternal chest pain, absolute dysphagia and spoke with difficulty. He was not inebriated and denied a background history of psychiatric or neurological illness.

He looked anxious and there was drooling of saliva. His vital signs were stable except for tachycardia. His neck extension was restricted. There was no oedema or crepitus noted at the neck or chest region. Auscultation of the lungs did not reveal any added sounds. The chest radiograph showed the head of the toothbrush lying at the level of the left bronchus (Figure $1)$.

Correspondence: G P U P de Silva

E-mail: upulonline@gmail.com

(i) https://orcid.org/0000-0003-2508-3765

Received: 26-02-2020 Accepted: 28-03-2021

DOI: http://doi.org/10.4038/sljs.v39i1.8762

The Sri Lanka Journal of Surgery 2021; 39(1): 67-69

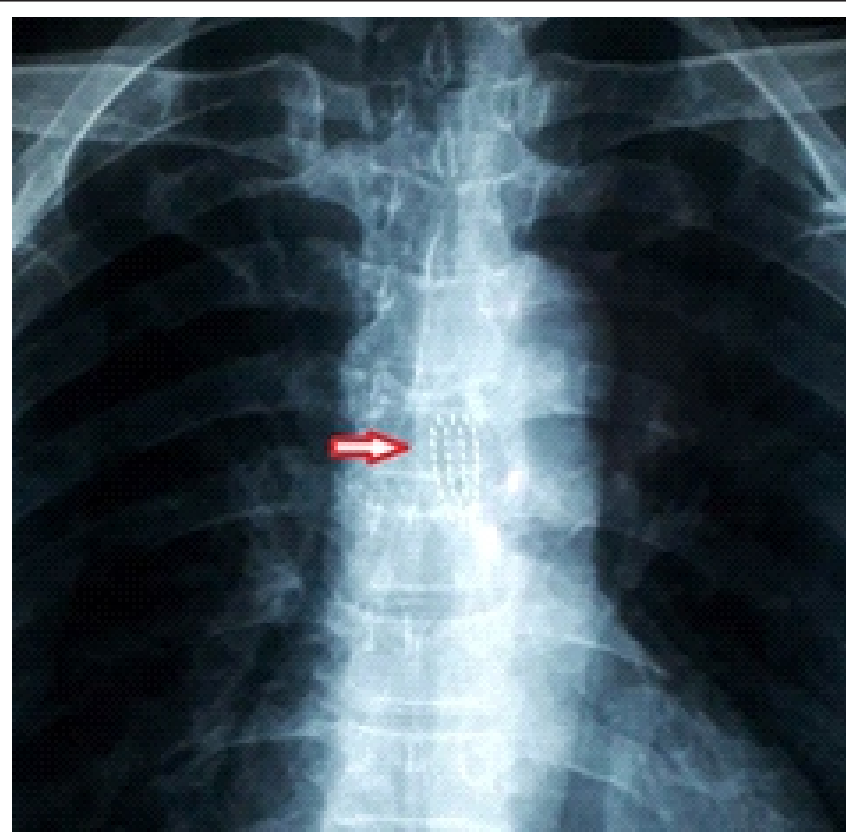

Figure 1. Chest radiograph showing head of the toothbrush

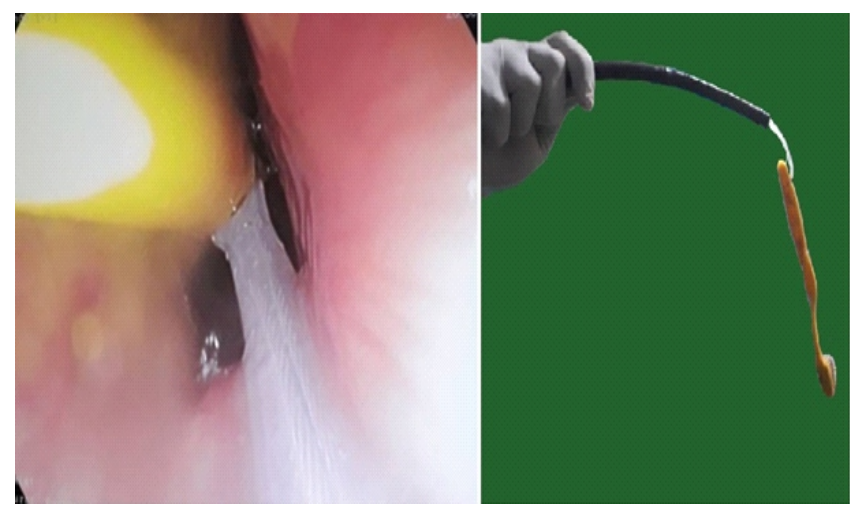

Figure 2. Removal of toothbrush with a snare loop

Emergency upper gastrointestinal endoscopy was carried out under deep sedation. The handle of the brush was seen just distal to the upper oesophageal sphincter. It was removed using a snare loop successfully (Figure 2 ). The recovery was uneventful and the patient has been discharged on the second post-procedure day.

\section{Discussion}

Upper gastrointestinal foreign bodies are reported commonly among children between 6 months to 3 years. Among the 
adults, fish bones and dentures are the commonly reported foreign bodies [2]. However, accidental ingestion of non-food objects in adults are rare and precipitated by neurological, psychiatric illnesses or alcohol intoxication [1].

Approximately 80-90\% out of upper gastrointestinal foreign bodies pass without any intervention, $10-20 \%$ require endoscopic removal and about $1 \%$ necessitate surgical removal. Impaction generally occurs at physiological narrowing and angulations. There are 4 narrowings at the oesophagus namely, upper oesophageal sphincter, level of the aortic arch, level of the left main bronchus (seen in this patient), and lower oesophageal sphincter. Pylorus, ileocecal valve and anus are the other physiological narrowing of the gastrointestinal tract. The duodenal sweep is the typical site for physiological angulation [3]. Foreign bodies longer than $5-10 \mathrm{~cm}$ are less likely to pass through the lower oesophagal sphincter and difficult to remove through the upper oesophagal sphincter [5].

Foreign bodies impacted in the oesophagus have the risk of complications including pressure necrosis, perforation, mediastinitis, retropharyngeal abscess and aspiration. The risk of developing a complication is increased by 14.1 times when the foreign body is impacted in the oesophagus for more than 24 hours. Also, the feasibility of successful removal is reduced with the delay [4].

Therefore therapeutic endoscopy should be arranged within 24 hours. However, the presence of features of complete oesophageal obstruction denoted by the inability to swallow saliva as in our patient warrants emergency endoscopic removal at least within 6 hours (preferably within 2 hours) since the higher risk for aspiration and aforementioned complications [2]. Our patient had an uneventful, early recovery and had been discharged on the following day, as we were able to perform the therapeutic endoscopy within few hours of the admission.

Radiological evaluation in suspected foreign body ingestion includes the x-rays of the neck, chest and abdomen to determine the size, site of impaction and configuration. This will further detect the presence of complications including pneumomediastinum, pneumoperitoneum, subcutaneous emphysema and aspiration [2]. Even though plastic objects are not regularly identified on X-rays due to their radiolucent nature, we were able to identify the head of the toothbrush on the $\mathrm{X}$-ray chest posteroanterior view (Figure 1). Lateral view of chest X-ray aids to locate the position of radio-opaque foreign body and to identify the presence of extraluminal air suggestive of oesophageal perforation [3].
Airway protection was a major concern as the high risk of aspiration due to inadequate fasting and proximal oesophageal location of the foreign body [2]. However, endotracheal intubation was difficult in our patient owing to apprehended position with limited neck extension.

The method of retrieval is decided by the size and configuration of the ingested foreign body. Polypectomy snare or Dormia basket is recommended for the removal of lengthy foreign bodies. The object must be $\backslash$ grasped at the end to facilitate successful removal since grasping near the centre would turn the object transversely hindering it from being pulled through the oesophagus. Before performing the therapeutic endoscopy, the most appropriate retrieval method can be determined by practising grasping an object similar to the ingested foreign body using different accessories $[2,5]$.

\section{Conclusions}

Oesophageal impaction of accidentally ingested non-food objects is rare among adults with intact sensorium. The spontaneous passage of long and stiff foreign objects similar to toothbrushes is unlikely and necessitate urgent therapeutic endoscopy to prevent complications including necrosis and perforation of oesophagus, mediastinitis, retropharyngeal abscess and aspiration.

All authors disclose no conflict of interest. The study was conducted in accordance with the ethical standards of the relevant institutional or national ethics committee and the Helsinki Declaration of 1975, as revised in 2000 .

\section{References}

1. Wyllie R. Foreign bodies in the gastrointestinal tract. Curr Opin Pediatr. 2006 Oct;18(5):563-4.

doi: 10.1097/01.mop.0000245359.13949.1c. PMID: 16969173.

2. Birk M, Bauerfeind P , Deprez P H, Häfner M, Hartmann D , Hassan C, Hucl T, Lesur G, Aabakken L, Meining A. Removal of foreign bodies in the upper gastrointestinal tract in adults: European Society of Gastrointestinal Endoscopy (ESGE) Clinical Guideline. Endoscopy. 2016 May;48(5):489-96. doi:10.1055/s-0042-100456. Epub 2016 Feb 10. PMID: 26862844.

3. Ambe P, Weber SA, Schauer M, Knoefel WT. Swallowed foreign bodies in adults. Dtsch Arztebl Int. 2012 Dec;109(50):869-75. doi: 10.3238/arztebl.2012.0869. Epub 2012 Dec 14. PMID: 23293675; PMCID: PMC3536040.

4. Loh KS, Tan LK, Smith JD et al. Complications of foreign bodies in the esophagus. Otolaryngol Head Neck Surg 2000; 123: 613-616.

5. Pfau PR.Removal and management of esophageal foreign bodies. Tech Gastrointest Endosc 2014; 16: 32-39 


\section{Learning Points:}

- Accidental ingestion of a toothbrush is rare.

- Therapeutic endoscopy is warranted within 24 hours for oesophageal foreign bodies to prevent sinister complications.

- Emergency endoscopic removal at least within 6 hours (preferably within 2 hours) if the features of complete oesophageal obstruction are present. 